JOURNAL OF THE

AMERICAN MATHEMATICAL SOCIETY

Volume 10, Number 3, July 1997, Pages 495-512

S 0894-0347(97)00215-4

\title{
THE TOPOLOGICAL UNIQUENESS OF COMPLETE ONE-ENDED MINIMAL SURFACES AND HEEGAARD SURFACES IN $\mathbb{R}^{3}$
}

\author{
CHARLES FROHMAN AND WILLIAM H. MEEKS III
}

\section{INTRODUCTION}

In this paper we shall prove two fundamental theorems on the topological uniqueness of certain surfaces in $\mathbb{R}^{3}$. The first of these theorems, which will depend on the second theorem, shows that a properly embedded minimal surface in $\mathbb{R}^{3}$ with one end is unknotted. More precisely,

Theorem 1.1. Two properly embedded one-ended minimal surfaces in $\mathbb{R}^{3}$ of the same genus are ambiently isotopic. ${ }^{1}$

Theorem 1.1 was conjectured by Frohman [10] who proved it in the case that the surfaces are triply-periodic. Earlier Meeks [19] proved the theorem in the case of finite genus. In this case the only known examples are the plane, the helicoid and a recent example of Hoffman, Karcher and Wei [16]. However, the collection of properly embedded minimal surfaces of infinite genus and one end is extremely rich. One reason for this is that most classical examples of these surfaces are doublyperiodic (i.e., they are invariant under translation in two independent directions) and in this case, a result of Callahan, Hoffman, and Meeks (Corollary 2 in [3]) states that the surface has one end and infinite genus, when it is not a plane. This result and Theorem 1.1 have the following corollary.

Corollary 1.1. Any two properly embedded nonplanar minimal surfaces in $\mathbb{R}^{3}$ that are invariant under at least two linearly independent translations are ambiently isotopic.

Essential in understanding the uniqueness theorems in this paper is the concept of a Heegaard surface in a noncompact three-manifold, which generalizes the usual notion of a Heegaard surface $M$ in a closed three-manifold $N^{3}$. Recall that $M$ is called a Heegaard surface if it separates $N^{3}$ into two genus- $g$ handlebodies where $g$

\footnotetext{
Received by the editors February 1, 1991 and, in revised form, June 1, 1995.

1991 Mathematics Subject Classification. Primary 57N12, 53A10.

This research was supported by the National Science Foundation grant DMS-8701736.

The research described in this paper was supported by research grant DE-FG02-86ER250125 of the Applied Mathematical Science subprogram of the Office of Energy Research, U.S. Department of Energy, and National Science Foundation grants DMS-8900285 and DMS-9505101.

${ }^{1}$ Two surfaces in $\mathbb{R}^{3}$ are ambiently isotopic if and only if there exists a one-parameter family of diffeomorphisms of $\mathbb{R}^{3}$ taking one surface to the other.
} 


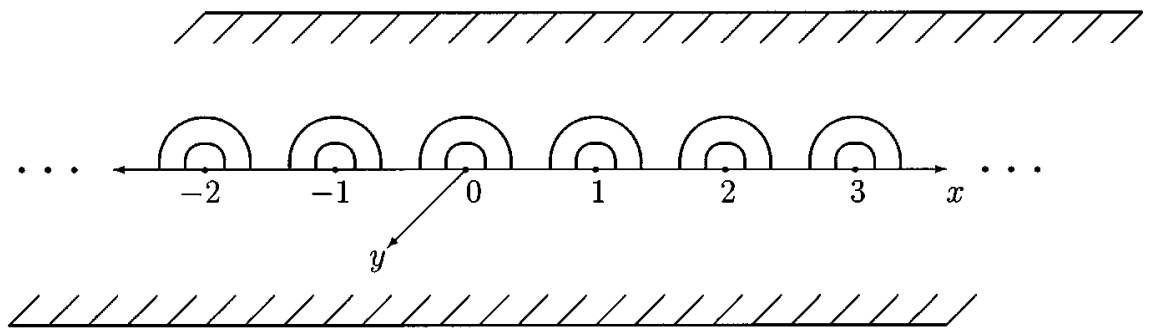

FiguRE 1

is the genus of $M$. (A handlebody of genus $g$ is also frequently referred to in the literature as a solid $g$-holed torus, a $g$-holed doughnut or a pretzel of genus $g$. Every closed three-manifold $N^{3}$ contains a Heegaard surface, so $N^{3}$ can be constructed by glueing two handlebodies together along their boundary (see [14] for details)).

Heegaard surfaces in $N^{3}$, which thereby yield "Heegaard splittings" of $N^{3}$ into handlebodies, play a fundamental role in classification questions in three dimensional topology. We refer the interested reader to $[1,2,4,10,9,12,14,18,19,25,31]$ for some of the theorems on Heegaard surfaces and the application of these theorems to geometry.

Noncompact three-manifolds such as $\mathbb{R}^{3}$ fail to have compact Heegaard surfaces. However, there is a natural notion of Heegaard surface for these manifolds where the surface is allowed to be noncompact. (See Section 2 for the precise definition.) We say that a properly embedded surface $M$ is a Heegaard surface in $\mathbb{R}^{3}$ if the closures of each of the two components of $\mathbb{R}^{3}-M$ are handlebodies. ${ }^{2}$ We will show that these handlebodies are each diffeomorphic to the three-manifold constructed as follows. Attach $g$ trivial one-handles to the closed lower halfspace $H$ in $\mathbb{R}^{3}$ where $g$ is the genus of $M$, possibly infinite. When $g=\infty$, this attaching of handles on $H$ can be performed on neighborhoods of the integer points on the $x$-axis contained in $\partial H$ to obtain a one-periodic Heegaard surface in $\mathbb{R}^{3}$ (see Figure 1). Note that this implies that a Heegaard surface in $\mathbb{R}^{3}$ has one end.

Our second main theorem is

Theorem 1.2. Heegaard surfaces of the same genus in $\mathbb{R}^{3}$ are ambiently isotopic. Equivalently, given two diffeomorphic Heegaard surfaces in $\mathbb{R}^{3}$, there exists a diffeomorphism of $\mathbb{R}^{3}$ that takes one surface to the other surface.

Theorem 1.2 will be proved at the end of Section 2 .

We will prove in Section 3 that a properly embedded one-ended minimal surface in $\mathbb{R}^{3}$ is a Heegaard surface. This result, together with Theorem 1.2, will prove Theorem 1.1.

The one-skeletons of different triangulations of $\mathbb{R}^{3}$ are not in general homeomorphic. However, since the boundary surfaces of their regular neighborhoods are Heegaard surfaces of infinite genus, Theorem 1.2 yields

Corollary 1.2. The regular neighborhoods of the one-skeletons of any two triangulations of $\mathbb{R}^{3}$ are ambiently isotopic.

${ }^{2} \mathrm{~A}$ three-manifold with boundary is a handlebody if it is homeomorphic to a closed regular neighborhood of a properly embedded one-dimensional CW-complex in $\mathbb{R}^{3}$. 


\section{Topological uniqueness of one-Ended HeEgaArd surfaces}

There is a subtle point related to the proof of Theorem 1.2 that we would like to point out to the reader. It follows immediately from the work of Frohman in [10] that a Heegaard surface of infinite genus in $\mathbb{R}^{3}$ contains an infinite number of disjoint trivial handles. (See Figure 3 for a picture of a trivial handle.) The main difficulty in proving Theorem 1.2 is to show that such a collection of disjoint trivial handles can be chosen to be a proper subset of $\mathbb{R}^{3}$. This subtle properness problem will be handled through the use of a compact exhaustion of $\mathbb{R}^{3}$.

Before proceeding to the proof of Theorem 1.2, we develop some basic notation, definitions and topological constructions that will be used in the analysis of Heegaard splittings of $\mathbb{R}^{3}$.

Let $M$ and $N$ be manifolds with boundary. A continuous map $f: M \rightarrow N$ is called proper if

1. For every compact set $K \subset N$, the set $f^{-1}(K)$ is compact, and

2. $f(\partial M)=f(M) \cap \partial N$.

We require all submanifolds to be topologically locally flat. We use the notation $\bar{A}$ to denote the closure of a set $A$.

We seek to generalize the notions of handlebody and compressionbody (to be defined) to include noncompact objects. A space $B$ is a handlebody if there exist a one-dimensional $C W$-complex $X$ that is properly embedded in $\mathbb{R}^{3}$ and a closed regular neighborhood, $N(X)$, that is homeomorphic to $B$. The genus of the handlebody $B$ is the genus of $\partial B$. If $B$ is compact, then, up to homeomorphism, $B$ is classified by its genus. Let $M$ and $N$ be three-manifolds with boundary. The boundary connect sum of $M$ and $N$ is the three-manifold $M \# N$ obtained by identifying a disk $D_{1}$ in the boundary of $M$ with a disk $D_{2}$ in the boundary of $N$.

A compressionbody is a space that can be constructed as follows. Let $F$ be a compact orientable surface with or without boundary. If $F$ has genus zero, we require that $\partial F$ be nonempty. Let $I$ denote the unit interval $[0,1]$. Let $B$ be a handlebody as above and let $D_{1}$ be a disk in $\partial B$. Let $D_{2}$ be a disk in $F \times$ $\{0\}$. The space $H=B \#(F \times I)$, where we take the sum along $D_{1}$ and $D_{2}$, is a compressionbody. The surface $\overline{\left(\partial B-D_{1}\right)} \cup \overline{\left(\partial F \times\{0\}-D_{2}\right)}$ will be called the distinguished surface of $H$. We can more generally define a compressionbody to be the boundary connect sum of a handlebody with a finite number of $F \times I$ where the surfaces $F$ are compact, and if some $F$ has genus zero then we require that $F$ have boundary. The restriction that the surfaces of genus 0 have boundary is to guarantee that compressionbodies have trivial second homotopy group.

Let $X$ be a topological space. If $K$ is a compact subset of $X$, then define $n(K)$ to be the number of connected components of $X-K$ that have noncompact closure. The number of ends of $X$, which we denote $e(X)$, is the supremum of $n(K)$ taken over the compact subsets of $X$. It is easy to check that $\mathbb{R}^{n}$ has two ends for $n=1$ and one end for $n>1$. The number of ends of a compressionbody $H$ is equal to the number of ends of its distinguished surface. The number of ends of a topological space can be computed as the rank of the 0-dimensional term of a cohomology theory [28]. There is an inequality relating the number of ends of a space $X$ to the number of ends of a pair of subspaces $X_{1}, X_{2}$ and their intersection $X_{1} \cap X_{2}$, such that $X=X_{1} \cup X_{2}$, that comes from a Mayer-Vietoris sequence. Specifically, if $\left(X, X_{1}, X_{2}\right)$ is an excisive triad, then $e(X) \geq e\left(X_{1}\right)+e\left(X_{2}\right)-e\left(X_{1} \cap X_{2}\right)$. 


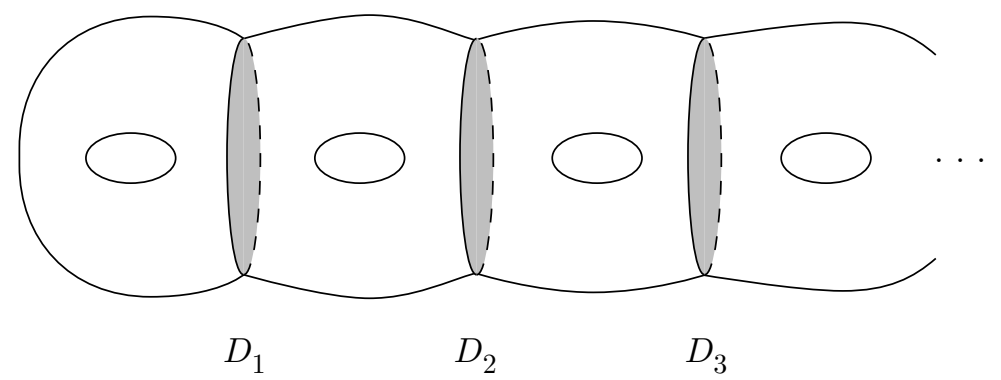

FiguRe 2

A simple closed curve $\kappa$ in a surface $F$ is essential if $\kappa$ is not the boundary of a disk embedded in $F$. A meridian disk $D$ for a handlebody $B$ is a properly embedded disk so that $\partial D$ is an essential simple closed curve in $\partial B$. A system of meridian disks is a disjoint union of disks $D_{i}$ that has been properly embedded in $B$ so that each disk in the family is a meridian disk. Let $B$ be a handlebody of infinite genus having one end. A standardizing sequence for $B$ is a system of meridian disks $\left\{D_{i}\right\}$ for $B$ where the index $i$ ranges over the positive integers, and the disk $D_{i}$ cuts $B$ into a handlebody of genus $i$ that contains all $D_{j}$ with $j<i$ and an unbounded component. (See Figure 2.) Every handlebody $B$ with one end and infinite genus has a standardizing sequence. From this it follows that up to homeomorphism there is only one handlebody of infinite genus having one end.

Definition 2.1. Let $M$ be a three-manifold and let $F_{1}$ and $F_{2}$ be compact surfaces in $\partial M$ so that $\partial M=F_{1} \cup F_{2}$ and $F_{1} \cap F_{2}=\partial F_{1}=\partial F_{2}$. A hollow Heegaard splitting of $\left(M, F_{1}, F_{2}\right)$ is a pair $\left(H_{1}, H_{2}\right)$ of compressionbodies $H_{1}$ and $H_{2}$ embedded in $M$ so that:

1. $H_{1} \cup H_{2}=M$;

2. $F=H_{1} \cap H_{2}$ is the distinguished surface of both $H_{1}$ and $H_{2}$;

3. $F$ is properly embedded in $M$;

4. $\partial F=F_{1} \cap F_{2}$;

5. $F_{i} \subset \partial H_{i}$.

We call $F$ the splitting surface associated to the hollow Heegaard splitting. If $H_{1}^{\prime}$ and $H_{2}^{\prime}$ are also hollow handlebodies, then we say that $\left(H_{1}, H_{2}\right)$ and $\left(H_{1}^{\prime}, H_{2}^{\prime}\right)$ of $\left(M, F_{1}, F_{2}\right)$ are topologically equivalent if there exists a homeomorphism $h: M \rightarrow M$ so that $h\left(H_{1}\right)=H_{1}^{\prime}$ and $h\left(H_{2}\right)=H_{2}^{\prime}$.

From our remarks about the ends of topological spaces, it can be seen that if $M$ is a three-manifold and $\left(H_{1}, H_{2}\right)$ is a Heegaard splitting of $M$, then $e(M) \geq$ $e\left(H_{1}\right)+e\left(H_{2}\right)-e\left(H_{1} \cap H_{2}\right)=e\left(H_{1} \cap H_{2}\right)$. Specifically, if $F$ is a splitting surface associated to a Heegaard splitting of $\mathbb{R}^{3}$, then $F$ has one end.

Let $\left(M, F_{1}, F_{2}\right)$ be a three-manifold with a hollow Heegaard splitting $\left(H_{1}, H_{2}\right)$, and let $F$ be the splitting surface associated with the Heegaard splitting. It is a consequence of Haken's lemma for hollow Heegaard splittings $[2,4]$ that if there is an incompressible sphere $S$ in $M$, then there is an incompressible sphere $S^{\prime}$ in $M$ that intersects $F$ in a single simple closed curve. The proof of this theorem is no different from the proof in the case where we require that all the manifolds involved be compact; the compactness of the sphere $S$ is all that is really necessary. 


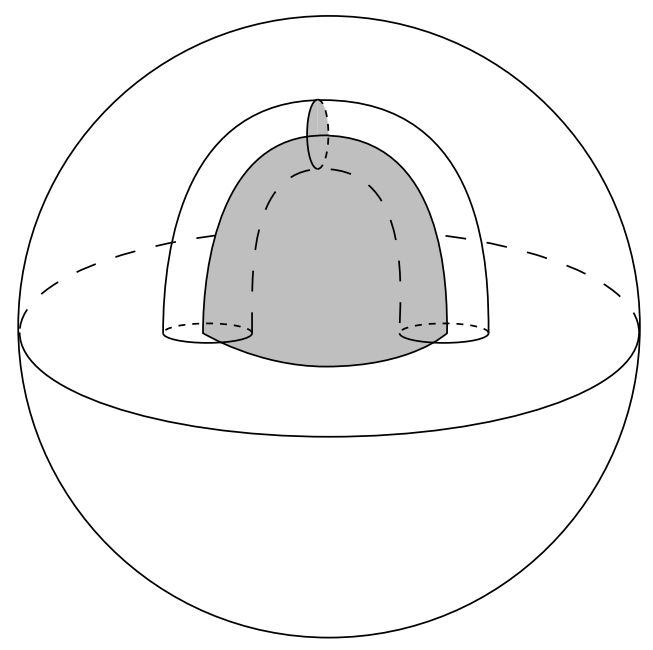

Figure 3 . The surface $T$ inside the ball $\mathbf{B}$

Let $\mathbf{B}$ denote the closed three-ball. Up to homeomorphism of $\mathbf{B}$, there is a unique compact genus-one surface $T$ with one boundary component, properly embedded in $\mathbf{B}$ so that there exist disks $D_{1}$ and $D_{2}$ embedded in $\mathbf{B}$ with $\partial D_{i}=D_{i} \cap T$ and such that $D_{1} \cap D_{2}$ consists of a single point of transverse intersection of $\partial D_{1}$ and $\partial D_{2}$ in $T$. The surface $T$ is the splitting surface associated to the unique (up to homeomorphism) genus-one Heegaard splitting with boundary of $\mathbf{B}$ having a single boundary component. The surface $T$ is pictured in Figure 3.

There is an operation on Heegaard splittings called stabilization. Let $F$ be the splitting surface associated to a Heegaard splitting of the three-manifold $M$. Let $\mathbf{B}$ be a ball embedded in $M$ that intersects $F$ in a single disk $D$. Let $F^{\prime}$ be a surface obtained from $F$ by removing the disk $D$ and replacing it with a surface $T$ as above. The surface $F^{\prime}$ is the splitting surface associated with a new Heegaard splitting $\left(H_{1}^{\prime}, H_{2}^{\prime}\right)$ of $M$. We call the new Heegaard splitting the result of stabilizing the old Heegaard splitting. This operation is uniquely defined up to homeomorphism. It is a theorem of Reidemeister and Singer [30] that any two Heegaard splittings of a closed three-manifold $M$ become topologically equivalent after each is stabilized a finite number of times.

Let $M$ be a compact three-manifold with boundary that is the result of removing open balls from $S^{3}$. Let $F_{1}$ and $F_{2}$ be surfaces in $\partial M$, so that $F_{1} \cup F_{2}=\partial M$, $F_{1} \cap F_{2}=\partial F_{1}=\partial F_{2}$, and the intersection of $F_{i}$ with any boundary component of $M$ consists of a single disk. Any planar surface $P$ that is properly embedded in $M$ so that $\partial P=\partial F_{1}$ is the splitting surface associated with a Heegaard splitting with boundary of $\left(M, F_{1}, F_{2}\right)$. Furthermore, a simple argument shows that any two such splittings are topologically equivalent. It is a consequence of Waldhausen's theorem [31] that any other Heegaard splitting with boundary of $\left(M, F_{1}, F_{2}\right)$ is topologically equivalent to the result of stabilizing $P$ the correct number of times. Specifically, Heegaard splittings of $\left(M, F_{1}, F_{2}\right)$ are classified by their genus.

Assume that $M$ has one end. Let $\left(H_{1}, H_{2}\right)$ be a Heegaard splitting of $M$ with splitting surface $F$. We say that $\left(H_{1}, H_{2}\right)$ is infinitely reducible if there exists a proper family of disjoint balls $\left\{B_{i}\right\}_{i=1}^{\infty}$ in $M$ so that each $B_{i}$ intersects $F$ in a copy 
of the surface $T$ from Figure 3 . We call the balls $B_{i}$ reducing balls. The appellation comes from the fact that if we replace the surfaces $B_{i} \cap F$ with disks the resulting surface is still a Heegaard surface. If a Heegaard splitting is infinitely reducible then we have a great deal of latitude in arranging its intersection with compact submanifolds of $M$.

Proposition 2.1. Suppose $M$ has one end. Let $\left(H_{1}, H_{2}\right)$ and $\left(H_{1}^{\prime}, H_{2}^{\prime}\right)$ be infinitely reducible Heegaard splittings of $\left(M, F_{1}, F_{2}\right)$ with splitting surfaces $F$ and $F^{\prime}$ respectively. Suppose that $K$ is a compact codimension 0 submanifold of $M$. Suppose that $G_{1}, G_{2}$ is a partition of the boundary of $K$ so that $F \cap K$ and $F^{\prime} \cap K$ are splitting surfaces for $\left(K, G_{1}, G_{2}\right)$. Note that $F \cap \partial K=F^{\prime} \cap \partial K=\partial G_{1}$. Then there are surfaces $\Phi$ and $\Phi^{\prime}$ isotopic to $F$ and $F^{\prime}$ with $\Phi \cap K=\Phi^{\prime} \cap K$ the splitting surface of a Heegaard splitting of $\left(K, G_{1}, G_{2}\right)$.

Proof. The proof is achieved by a process we call "stealing handles from infinity". If we can show that we can isotopy $F$ to a surface $\Phi$ so that $\Phi \cap \partial K=F \cap \partial K$ and $\Phi \cap K$ is the result of stabilizing $F \cap K$ an arbitrary number of times, then the Reidemeister-Singer theorem will imply the desired result.

Since $K$ is compact there exist infinitely many reducing balls for $\left(H_{1}, H_{2}\right)$ that are disjoint from $K$. Let $B$ be such. Since a ball is a regular neighborhood of any point in its interior we may isotope $B$ so that it is a small ball centered at a point $p$ in $F$. Let $q$ be a point in $F \cap \stackrel{\circ}{K}$. Since $F$ is path connected there is an arc in $F$ joining $p$ and $q$. Effect an isotopy supported in a small regular neighborhood of this arc that drags the contents of $B$ to a small ball in $\stackrel{\circ}{K}$ centered at $q$. Call the resulting surface $\Phi$. It is clear that we can effect this isotopy so that $\Phi \cap K$ is the result of stabilizing $F \cap K$ once, with $\Phi \cap \partial K=F \cap \partial K$. Furthermore we can repeat this process an arbitrary number of times.

Given a Heegaard splitting of a noncompact manifold we can find an exhaustion of the manifold by compact submanifolds so that the intersection of the Heegaard splitting with the compact "pieces" is a Heegaard spliting.

Proposition 2.2. Let $\left(H_{1}, H_{2}\right)$ be a Heegaard splitting of $\left(M, F_{1}, F_{2}\right)$ with splitting surface $F$. There exists an exhaustion of $M$ by compact submanifolds $K_{i}, K_{0}=$ $\emptyset, K_{i} \subseteq \stackrel{\circ}{K}_{i+1}$ so that for all $i,\left(H_{1} \cap\left(K_{i}-\stackrel{\circ}{K}_{i-1}\right), H_{2} \cap\left(K_{i}-\stackrel{\circ}{K}_{i-1}\right)\right)$ is a Heegaard splitting of $K_{i}-\stackrel{\circ}{K}_{i-1}$ (with the obvious partition of $\partial\left(K_{i}-\stackrel{\circ}{K} i-1\right)$ ).

Proof. Since $H_{1}$ is compression body with preferred surface $F$ we can find an exhaustion $L_{i}$ of $H_{1}$ by compact submanifolds so that for all $i, L_{i}-\stackrel{\circ}{L}_{i-1}$ is a compression body with preferred surface $F \cap\left(L_{i}-\stackrel{\circ}{L}_{i-1}\right)$. Similarly we can find an exhaustion $J_{i}$ of $H_{2}$ so that the $J_{i}-\stackrel{\circ}{J}_{i-1}$ are compression bodies with preferred surface $F \cap\left(J_{i}-\stackrel{\circ}{J}_{i-1}\right)$. Let $K_{i}$ be the union of $J_{i}$ and $L_{i}$ along with collars of $J_{i}-L_{i}$ in $H_{1}$ and $L_{i}-J_{i}$ in $H_{2}$, chosen to intersect $J_{i} \cup L_{i}$ nicely. The exhaustion $K_{i}$ will have the desired property.

We call an exhaustion as above adapted to $\left(H_{1}, H_{2}\right)$.

Proposition 2.3. If $\left(H_{1}, H_{2}\right)$ and $\left(H_{1}^{\prime}, H_{2}^{\prime}\right)$ are infinitely reducible Heegaard splittings of $\left(M, F_{1}, F_{2}\right)$ with splitting surfaces $F$ and $F^{\prime}$ then there exists an exhaustion $\left\{L_{i}\right\}$ of $M$ so that after isotopies fixing $\partial M,\left\{L_{i}\right\}$ is adapted to both $\left(H_{1}, H_{2}\right)$ and $\left(H_{1}^{\prime}, H_{2}^{\prime}\right)$ and the intersections of $F$ and $F^{\prime}$ with the frontier of each $L_{i}$ coincide. 
Proof. Suppose that $\left\{K_{i}\right\}$ and $\left\{K_{i}^{1}\right\}$ are exhaustions of $M$ that are adapted to $\left(H_{1}, H_{2}\right)$ and $\left(H_{1}^{\prime}, H_{2}^{\prime}\right)$. By inductively selecting subsequences and renumbering we may assume that for all $i$,

$$
K_{i-1} \subseteq \stackrel{\circ}{K}_{i-1}^{\prime} \subseteq K_{i-1}^{\prime} \subseteq \stackrel{\circ}{K}{ }_{i}
$$

Choose the exhaustion $L_{i}$ so that

$$
K_{i-1} \subseteq \stackrel{\circ}{L}_{i} \subseteq L_{i} \subseteq K_{i}
$$

and

$$
K_{i-1}^{\prime} \subseteq \stackrel{\circ}{L}_{i} \subseteq L_{i} \subseteq K_{i}^{\prime}
$$

For instance the frontier of $L_{i}$ could be a pushoff of the frontier of $K_{i}$ into $K_{i}$. Partition the boundary of $L_{i}$ into $\left(G_{i}(i), G_{2}(i)\right)$ so that it coincides with $F_{1}, F_{2}$ on the overlap. There exist Heegaard splittings of $L_{i}-\stackrel{\circ}{K}{ }_{i-1}$ and $K_{i}-\stackrel{\circ}{L}_{i}$ so that the partition of the boundary restricted to the boundary of $L_{i}$ coincides with $\left(G_{1}(i), G_{2}(i)\right)$ and the partition of the boundary restricted to the boundary of $K_{i}$ coincides with the partition of the boundary of $K_{i}$ induced by $\left(H_{1}, H_{2}\right)$. Together these Heegaard splittings form a Heegaard splitting of $K_{i}-\stackrel{\circ}{K} \underset{i-1}{ }$ intersecting the boundary of $L_{i}$ in $\partial G_{1}(i)$. We can stabilize $F \cap K_{i}-\stackrel{\circ}{K}_{i-1}$ so that it is isotopic relative to its boundary to a surface having $\partial G_{1}(i)$ as its intersection with the boundary of $L_{i}$. By stealing handles from infinity we can isotope $F$ so that this is true. Still working with $\left(G_{1}(i), G_{2}(i)\right)$ we can do the same with $F^{\prime}$, that is after an isotopy $F^{\prime}$ intersects the boundary of $L^{\prime}$ in $\partial G_{1}(i)$. Furthermore we can do this so that $F \cap\left(K_{i}-\stackrel{\circ}{L}_{i}\right), F \cap\left(L_{i}-\stackrel{\circ}{K}_{i-1}\right), F^{\prime} \cap\left(K_{i}^{\prime}-\stackrel{\circ}{L}_{i}\right)$ and $F^{\prime} \cap\left(L_{i}-K_{i-1}^{\prime}\right)$ are all splitting surfaces. This means that $F \cap\left(L_{i}-\stackrel{\circ}{L}_{i-1}\right)$ and $F^{\prime} \cap\left(L_{i}-\stackrel{\circ}{L}_{i-1}\right)$ are splitting surfaces and $F \cap \partial L_{i}=F^{\prime} \cap \partial L_{i}$. Proceed inductively making sure that after some $N$, depending on $i$, if $n \geq N$, then the surface's intersection with $L_{i}$ is the same before and after the isotopies. This will imply that the process converges.

Theorem 2.1. Let $\left(H_{1}, H_{2}\right)$ and $\left(H_{1}^{\prime}, H_{2}^{\prime}\right)$ be infinitely reducible Heegaard splittings of $\left(M, F_{1}, F_{2}\right)$ with splitting surfaces $F$ and $F^{\prime}$. Then $F$ and $F^{\prime}$ are isotopic.

Proof. First apply Proposition 2.3 to find an exhaustion $\left\{L_{i}\right\}$ and proper isotopies of $F$ and $F^{\prime}$ so that $\left\{L_{i}\right\}$ is adapted to the isotoped Heegaard splittings. Then use Proposition 2.1 inductively on $L_{i}-\stackrel{\circ}{L}_{i}$ to isotope $F$ so that it coincides with $F^{\prime}$. We can use the fact that all the handles we are stealing from infinity can be taken from the only end representative to make sure that after some stage in the construction we are not disturbing $L_{j} \cap F$. Hence the process converges.

Once it has been established that infinite genus Heegaard splittings of $\mathbb{R}^{3}$ are infinitely reducible, then Theorem 1.2 from the introduction follows from Theorem 2.1. Let $F$ be a splitting surface for a Heegaard splitting of $\mathbb{R}^{3}$, let $\Gamma$ be a graph so that $F$ is a regular neighborhood of its boundary. Let $K$ be a compact subset of $\mathbb{R}^{3}$. From Proposition 2.2 we see that there is a compact set $L$ with $K \subset L$, so that $\overline{F-L}$ is a splitting surface for a Heegaard splitting of the complement of $L$. There exists a ball $B$ with $L \subset \operatorname{int}(B)$. Since $\Gamma$ has one end, there exists a cycle of $\Gamma$ outside the ball $B$. It is easy to find a ball $B^{\prime}$ that contains this cycle that does not intersect $B$. Therefore $\overline{F-L}$ is reducible [10]. This is enough to conclude that $F$ is infinitely reducible. Hence Theorem 1.2 follows. 


\section{Topological uniqueness of One-Ended complete minimal Surfaces}

In this section we will be studying the geometry and topology of the closure of the components of the complement of properly embedded surfaces in $\mathbb{R}^{3}$. These manifolds with boundary are almost-complete in the following sense.

Definition 3.1. A Riemannian $n$-manifold with boundary is called almost-complete if it is complete as a metric space with respect to the natural distance function induced from the infimum of the lengths of curves joining pairs of points in the manifold.

We will study the topology of a properly embedded minimal surface $M$ in $\mathbb{R}^{3}$ by showing that the closure of each component of $\mathbb{R}^{3}-M$ is a standard one-ended handlebody whenever $M$ has one end. When $M$ has finite topology, this was proved by Meeks [19]; the case of interest here will be when $M$ has one end and infinite genus.

Recall that a noncompact surface $\Sigma$ in a Riemannian manifold $N$ has least area if for any smooth compact subdomain $\Delta \subset \Sigma, \Delta$ is a surface of least area in $N$ with boundary $\partial \Delta$.

Lemma 3.1. Suppose $N$ is a connected, orientable, almost-complete Riemannian three-manifold with more than one boundary component. If $\partial N$ has nonnegative mean curvature with respect to the outward pointing normal, then $N$ contains a properly embedded, orientable, least-area minimal surface.

Proof. Suppose $\partial_{1}$ and $\partial_{2}$ are two components of $\partial M$. Choose an $\operatorname{arc} \delta$ in $N$ that joins a point $p \in \partial_{1}$ with a point of $\partial_{2}$. Let $\widetilde{\Sigma}_{1} \subset \widetilde{\Sigma}_{2} \subset \ldots$ be a smooth compact exhaustion of $\partial_{1}$ with $p \in \widetilde{\Sigma}_{1}$. Theorem 1 in [24] (together with the general regularity theory of area-minimizing currents in [13] and [29]) shows that $\partial \widetilde{\Sigma}_{i}$ is the boundary of a least-area surface $\Sigma_{i}$ in $N$ such that $\Sigma_{i}$ is homologous $\operatorname{rel}(\partial N)$ to $\widetilde{\Sigma}_{i}$ with $\mathbb{Z}_{2}$-coefficients. Note that $\Sigma_{i}$ is orientable since $\Sigma_{i} \cup \widetilde{\Sigma}_{i}$ is a boundary in $N$.

Since the surface $\Sigma_{i}$ is area-minimizing in the interior of $N$, for any ball $\mathbf{B}$ $\subset \operatorname{Int}(N)$, a simple replacement argument using portions of $\partial \mathbf{B}$ shows that $\operatorname{Area}\left(\mathbf{B} \cap \Sigma_{i}\right) \leq \frac{1}{2} \operatorname{Area}(\partial \mathbf{B})$. Similarly, if $\mathbf{B}$ is a smooth ball of geodesic radius $\varepsilon$ centered at a point in $\partial N$, then $\operatorname{Area}\left(\mathbf{B} \cap \Sigma_{i}\right) \leq \operatorname{Area}(\partial \mathbf{B})$. These estimates show that the family $\left\{\Sigma_{i}\right\}$ has uniform local area bounds. These area bounds are sufficient for applying the standard compactness and regularity theorems [29] of geometric measure theory which state that a subsequence $\Sigma_{i_{j}}$ of these surfaces converges smoothly on compact subsets of $N$ to a properly embedded least-area surface $\Delta$. (The property that a limit of area-minimizing surfaces is itself area-minimizing is well known and is proved in a similar context in the last paragraph of the proof of Theorem 3.1 in [25].) Note that each of the surfaces $\Sigma_{i}$ has odd intersection number with the $\operatorname{arc} \delta$ and so $\Delta \cap \delta \neq \emptyset$, which implies $\Delta$ is nonempty. This completes the proof of the lemma.

Theorem 3.1. Suppose $N$ is a connected, orientable, almost-complete, flat threemanifold. If $\partial N$ has nonnegative mean curvature with respect to the outward pointing normal, then either $\partial N$ is connected or $N$ is isometric to the Riemannian product $\Sigma \times[0, T]$ for some $T>0$ and for some complete totally geodesic surface $\Sigma$ in $N$. 


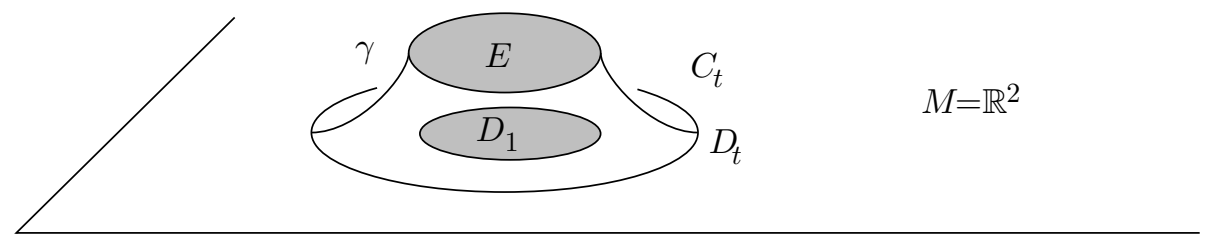

Figure 4

Proof. Suppose $N$ has at least two boundary components. For the moment, also assume that $N$ is simply connected. We shall prove the $N$ is isometric to $\mathbb{R}^{2} \times[0, T]$ for some $T>0$.

Let $M \subset N$ denote a properly embedded, stable, orientable, minimal surface whose existence is guaranteed in Lemma 3.1. By the work of do Carmo and Peng [5] or of Fischer-Colbrie and Schoen [7], we know that $M$ is totally geodesic. Since $N$ is simply connected, $M$ must be a plane in $N$. (When $N$ is simply connected, it isometrically submerses into $\mathbb{R}^{3}$ and so $M$ must be a plane.) After a translation of $M$ in $N$, we may assume that the distance from $M$ to $\partial N$ is zero. By the maximum principle, either $M \subset \partial N$ or else $M \cap \partial N=\emptyset$. Suppose for the moment that $M \cap \partial N=\emptyset$ and $\operatorname{dist}(M, \partial N)=0$ and identify $M$ with the plane $\mathbb{R}^{2}$.

Let $D_{t}$ be the disk of radius $t$ in $\mathbb{R}^{2}$ centered at the origin. Suppose $\operatorname{dist}\left(D_{1}, \partial N\right)$ $>\varepsilon$ and choose $\varepsilon<\frac{1}{4}$. Let $\gamma$ denote the circle of height $\varepsilon$ over $\partial D_{1}$. The curves $\gamma \cup \partial D_{1}$ bound a stable catenoid $C_{1}$ with $C_{1} \cap \partial N=\partial D_{1}$. (In our discussion of catenoids we are using the fact that some neighborhood of $M$ in $N$ isometrically embeds in $\mathbb{R}^{3}$.) Furthermore it is clear that, for $t$ close to $1, \gamma \cup \partial D_{t}$ is also the boundary of a stable catenoid $C_{t}$ and the family $C_{t}$ varies continuously with $t$. The maximum principle shows that the interiors of the one-parameter family of stable catenoids $C_{t}$ are always disjoint from $\partial N$ for $t \geq 1$ and for as long as $C_{t}$ is defined in $N$. It is clear that $C_{t}$ is defined for all $t \geq 1$ and $C_{t_{1}}$ lies above $C_{t_{2}}$ when $t_{1}>t_{2}$. Since the $C_{t}$ are stable catenoids, they converge to a totally geodesic surface $C$ in $N$ with $\partial C=\gamma$ (see Figure 4).

Let $E$ be the flat disk with $\partial E=\gamma$. Then $C_{t} \cup E \cup D_{t}$ bounds a compact region $R \subset N$. The regions $R_{t}$ converge to a slab of width $\varepsilon$ that is isometrically embedded in $N$ and one of its boundary planes is $M$. These same arguments show that $M$ is also the boundary of another slab of width $\varepsilon$ on its other side. Hence, the distance from $M$ to $\partial N$ is positive, a contradiction of our earlier assumption that $M \cap \partial N=\emptyset$ and $\operatorname{dist}(M, \partial N)=0$.

If $M \subset \partial N$, a simple modification of the arguments given above in the case $M \cap \partial N=\emptyset$ shows that the boundary component $M$ can be continuously translated away from itself until it touches another boundary component of $N$ at which point it equals that boundary component. Thus, we see that $N$ is isometric to $\mathbb{R}^{2} \times[0, T]$ for some $T>0$.

If $N$ is not simply connected, then by the previously considered case, the simply connected Riemannian cover of $N$ is isometric to $\mathbb{R}^{2} \times[0, T]$ for some $T>0$. Since the covering transformations are isometries and $N$ has more than one end, $N$ must be isometric to $\Sigma \times[0, T]$ where $\Sigma$ is a flat orientable surface.

Remark 3.1. The main idea in the proof of Theorem 3.1 (of using limits of catenoids) was first used by Hoffman and Meeks in the proof of their Halfspace Theorem that 
states that a proper nonplanar minimal surface in $\mathbb{R}^{3}$ that is contained in a halfspace is a plane. The Halfspace Theorem and Theorem 3.1 are examples of maximum principles at infinity for proper minimal surfaces in a flat three-manifolds. (See [21] for a useful and general version of this type of maximum principle.)

Corollary 3.1. Suppose $N$ is an almost-complete flat three-manifold whose boundary has nonnegative mean curvature with respect to the outward pointing unit normal. If the map $\pi_{1}(\partial N) \rightarrow \pi_{1}(N)$ is not surjective, then $N$ or a 2-sheeted covering space of $N$ is a Riemannian product.

Proof. If $\pi_{1}(\partial N) \rightarrow \pi_{1}(N)$ is not surjective, then the universal cover $\widetilde{N}$ satisfies the hypotheses of Theorem 3.1 and $\partial \widetilde{N}$ is not connected. Hence, $\widetilde{N}$ is isometric to $\mathbb{R}^{2} \times[0, T]$ for some $T>0$. The corollary follows immediately from this fact.

Corollary 3.2. Suppose $M$ is a properly embedded minimal surface in $\mathbb{R}^{3}$ and $N$ is the closure of a component of $\mathbb{R}^{3}-M$. Then the interior of $N$ is an open handlebody and the induced map $\pi_{1}(M) \rightarrow \pi_{1}(N)$ is surjective.

Proof. It follows from Theorem 3 in [19] that $\operatorname{Int}(N)$ is an open handlebody. The second statement follows immediately from Corollary 3.1 .

Remark 3.2. Corollary 3.2 was independently proved by Joel Hass (personal communication).

When a properly embedded minimal surface $M \subset \mathbb{R}^{3}$ has one end, we can prove even more concerning the topology of the closed complements of $M$.

Theorem 3.2. Suppose $M$ is a properly embedded one-ended minimal surface in $\mathbb{R}^{3}$ and $N$ is the closure of one of the components of $\mathbb{R}^{3}-M$. Then $N$ is a one-ended handlebody. In particular, $M$ is a Heegaard surface in $\mathbb{R}^{3}$.

The next theorem, which is stated in the Introduction, is a simple consequence of the above theorem and Theorem 1.2.

Theorem 1.1. Two properly embedded one-ended minimal surfaces in $\mathbb{R}^{3}$ of the same genus are ambiently isotopic.

Proof of Theorem 3.2. Let $M \subset \mathbb{R}^{3}$ be a properly embedded minimal surface with one end. If $M$ has finite genus, then it was proved in [19] that $M$ is a Heegaard surface. Assume now that $M$ has infinite genus. We shall show that the closure $N$ of a component of $\mathbb{R}^{3}-M$ must be a handlebody. This will imply $M$ is a Heegaard surface in $\mathbb{R}^{3}$ and Theorem 3.2 will then follow from Theorem 1.2.

Since $\operatorname{Int}(N)$ is an open handlebody by Corollary 3.2 , to prove $N$ is a handlebody it suffices to prove that there exists a proper standardizing sequence $\mathcal{D}=\left\{D_{1}, D_{2}, \ldots\right\}$ in $N$ satisfying,

1. For all $k, \partial D_{k}=D_{k} \cap N$ represents a homotopically nontrivial curve in $N$;

2. $D_{k}$ separates $N$ into a compact component $N_{k}$ and a noncompact component where $\bigcup_{i<k} D_{i} \subset N_{k}$;

3. $\bigcup_{k} N_{k}=\bar{N}$.

The first step in the proof is to construct an infinite sequence of auxiliary minimal surfaces $\left\{\Sigma_{1}, \Sigma_{2}, \ldots\right\}$ that satisfy some of the properties required for the disks in $\mathcal{D}$. First choose a compact exhaustion $\widetilde{\Sigma}_{1} \subset \widetilde{\Sigma}_{2} \subset \cdots$ of $M=\partial N$ where $\partial \widetilde{\Sigma}_{i}$ is a simple closed curve. Since $\partial N$ has nonnegative mean curvature and $\partial \widetilde{\Sigma}$ bounds 
in $N, \partial \widetilde{\Sigma}_{i}$ is the boundary of a compact least-area surface $\Sigma_{i}$ in $N$ homologous $\operatorname{rel}(\partial N)$ to $\widetilde{\Sigma}_{i}$ with $\mathbb{Z}_{2}$-coefficients (see Theorem 1 in [24] and also [29]). Note $\Sigma_{i}$ is orientable since $\Sigma_{i} \cup \widetilde{\Sigma}_{i}$ bounds.

We next show that the $\Sigma_{i}$ can be chosen so that $\bigcup_{i} \Sigma_{i}$ is a properly embedded submanifold of $\mathbb{R}^{3}$. If the sequence $\Sigma_{i}$ is not eventually disjoint from every compact subset of $\mathbb{R}^{3}$, then, after choosing a subsequence, there exist points $p_{i} \in \Sigma_{i}$ with $p_{i} \rightarrow p$ for some point $p \in N$. Since $\Sigma_{i}$ is a $\mathbb{Z}_{2}$-area-minimizing surface, a subsequence $\Sigma_{i_{j}}$ of these surfaces converges smoothly on compact subsets of $N$ to a properly embedded least-area surface $\Delta$ (see the proof of Lemma 3.1 for this convergence argument). Note that $\Delta$ is nonempty since $p \in \Delta$. But $\Delta$ is a complete stable orientable minimal surface in $\mathbb{R}^{3}$ and hence every component of $\Delta$ is a plane ([5] or [7]). The Halfspace Theorem in [17] states that a properly immersed minimal surface in $\mathbb{R}^{3}$ that is contained in halfspace is a plane but $M$ is not a plane. This contradiction shows that the sequence $\Sigma_{i}$ eventually leaves every compact subset of $\mathbb{R}^{3}$. It follows, by choosing a subsequence, that we can also choose $\left\{\Sigma_{i}\right\}$ so that these surfaces are pairwise disjoint. This completes the construction of the family $\left\{\Sigma_{i}\right\}$.

Let $R_{i}$ denote the noncompact region of $N$ bounded by $\Sigma_{i} \cup\left(M-\widetilde{\Sigma}_{i}\right)$. Note that $R_{i+1} \subset R_{i}$. We will construct the required sequence of disks $\mathcal{D}$ by showing that for each $i$, there exists a disk $D_{i} \subset R_{i}$ with $\partial D_{i}=\left(D_{i} \cap \partial R_{i}\right) \subset M$ and such that $\partial D_{i}$ is homologous in $\overline{M-\widetilde{\Sigma}_{i}}$ to the curve $\partial \Sigma_{i}$. It is straightforward to check that such a collection of disks contains a reindexed subcollection with the required Properties 1-3 for the collection $\mathcal{D}$. We first prove the existence of the disk $D_{1}$.

The two curves $\partial \Sigma_{1} \cup \partial \Sigma_{i}$ bound a subdomain $M_{i}$ of $M$. If either $\partial \Sigma_{1}$ or $\partial \Sigma_{i}$ is homotopically trivial in $R_{1}$, then Dehn's Lemma [14] shows that this curve is the boundary of an embedded disk $D_{1}$ in $R_{1}$. Hence, we may assume that $\partial \Sigma_{1}$ and $\partial \Sigma_{i}$ are both homotopically nontrivial in $R_{1}$. The loop theorem [14] implies that there exists a pairwise disjoint collection of disks in $R_{1}$ with boundary curves in $M_{i}$ such that after performing a surgery of $M_{i}$ along these disks the resulting surgered surface $\widehat{M}_{i}$ is incompressible in $R_{1}$. (Recall that performing a surgery of $M$ along a disk $E$ with $\partial E \subset M$ means that for some product neighborhood, $P(E) \subset R_{1}$, we replace $P(E) \cap M$ by the two disks $\partial P(E)-(P(E) \cap M))$. Let $M_{i}^{\prime}$ denote the component of $\widehat{M}_{i}$ that contains $\partial \Sigma_{1}$. Suppose for the moment that $\partial \Sigma_{i} \not \subset \partial M_{i}^{\prime}$ for some $i$. In this case let $D_{1}$ be the boundary connected sum of the disks $M_{i}^{\prime} \cap \operatorname{Int}(N)$ along pairwise disjoint arcs in $M_{i}^{\prime} \cap M_{i}$. Note that $D_{1}$ is homologous rel $\left(\partial R_{1}-\operatorname{Int}\left(\Sigma_{1}\right)\right)$ in $R_{1}$ to $\Sigma_{1}$. Hence, we may assume that the incompressible surface $M_{i}^{\prime}$ has boundary $\partial \Sigma_{1} \cup \partial \Sigma_{i}$.

Replace the incompressible surface $M_{i}^{\prime}$ by an embedded minimal surface $\widetilde{M}_{i}$ of least area in $R_{1}$ in the isotopy (or homotopy class) of $M_{i}^{\prime}$ (see [8] or [22]). We will prove in Assertion 3.1 that a subsequence $\widetilde{M}_{i j}$ of the $\widetilde{M}_{i}$ converges smoothly on compact subsets of $\mathbb{R}^{3}$ to a smooth, connected, noncompact, properly embedded, stable, orientable, minimal surface $\Sigma \subset R_{1}$ with $\partial \Sigma=\partial \Sigma_{1}$. Assume for the moment that Assertion 3.1 holds and $\Sigma$ is given.

We first check that $\Sigma$ is incompressible. Let $\alpha$ be a simple closed curve in $\Sigma$ that bounds a disk $D$ in $R_{1}$. Since $\widetilde{M}_{i_{j}}$ converges smoothly to $\Sigma$ on compact subsets of $R_{1}$, for $j$ large we can lift $\alpha$ to a nearby $\alpha_{j} \subset \widetilde{M}_{i_{j}}$ and $\alpha_{j}$ is homotopically trivial in $R_{1}$. Since $\widetilde{M}_{i_{j}}$ is incompressible, $\alpha_{j}$ is the boundary of a minimal disk $D_{j} \subset \widetilde{M}_{i_{j}}$ 
contained in the convex hull of $\alpha_{j}$. Note however that a subsequence of the leastarea minimal disks $\left\{D_{j}\right\}$ converges to a least-area disk $D^{\prime} \subset \Sigma$ with $\alpha=\partial D^{\prime}$ (see [23] for this type of compactness argument). This proves $\Sigma$ is incompressible.

It follows from [6] (or Theorem 2.1 in [25]) that $\Sigma$ has finite total curvature (since $\Sigma$ is complete, orientable, stable and $\partial \Sigma$ is compact) and hence, $\Sigma$ has finite topology. Since $\Sigma$ has finite genus and $\partial R_{1}$ has infinite genus we conclude that $\Sigma \not \subset \partial R_{1}$. The maximum principle implies $\Sigma \cap \partial R_{1}=\partial \Sigma$. Since $\Sigma$ has finite total curvature, its ends are asymptotic to planes and catenoids (see [27]). Let $B(r)$ denote the ball of radius $r$ centered at the origin. For large values of $r$, the sphere $\partial B(r)$ intersects $\Sigma$ almost orthogonally in a finite collection of curves that are geometrically close to a fixed great circle. In particular, for some fixed large value of $r, \Sigma$ separates $\mathbb{R}^{3}-B(r)$ into a finite number of components, exactly one of which contains the end of $M \cap\left(\mathbb{R}^{3}-B(r)\right)$. Since $M$ has one end, $M \cap \overline{\left(\mathbb{R}^{3}-B(r)\right)}$ has exactly one noncompact component, and therefore the convex hull property implies that $M \cap\left(\mathbb{R}^{3}-B(r)\right)$ is connected. Hence, every component $\delta$ of $\Sigma \cap \partial B(r)$ bounds a disk in $\partial B(r)$ that is disjoint from $M$ and therefore this disk is contained in $R_{1}$. But $\delta$ is homotopically nontrivial on $\Sigma$ and $\Sigma$ is incompressible in $R_{1}$. This contradiction proves the existence of $D_{1}$.

Replacing $\Sigma_{1}$ with $\Sigma_{i}$ and repeating the above argument proves the existence of a disk $D_{i} \subset R_{i}$ that is homologous to $\Sigma_{i}$ in $R_{i} \operatorname{rel}\left(\partial R_{i}-\operatorname{Int}\left(\Sigma_{i}\right)\right)$. It is straightforward to prove that the disks $\left\{D_{i}\right\}$ have the required properties, which completes the proof of the existence of the family $\mathcal{D}$. Thus, to complete the proof of Theorem 3.2, it remains only to prove the next assertion.

Assertion 3.1. A subsequence $\widetilde{M}_{i_{j}}$ of the surfaces $\widetilde{M}_{i}$ converges smoothly on compact subsets of $\mathbb{R}^{3}$ to a smooth, connected, noncompact, properly embedded, stable, orientable, minimal surface $\Sigma \subset R_{1}$ with $\partial \Sigma=\gamma=\partial \Sigma_{1}$.

Proof of Assertion 3.1. Suppose a subsequence $\widetilde{M}_{i_{j}}$ converges smoothly to a properly embedded minimal surface $\Sigma$ with boundary $\gamma$. Then each component of $\Sigma$ is a properly embedded stable minimal surface. If $\Sigma$ is not connected, then there is a component $\Delta$ of $\Sigma$ that is a properly embedded surface in $\mathbb{R}^{3}$ and has empty boundary. Since $\Delta$ separates $\mathbb{R}^{3}$, it is orientable. If $\Delta$ is unstable, then it contains a smooth compact subdomain $\widetilde{\Delta}$ that is unstable. Since there are subdomains $\widetilde{\Delta}\left(i_{j}\right) \subset \widetilde{M}_{i_{j}}$ that converge smoothly to $\widetilde{\Delta}$, the lowest eigenvalue of the Jacobi operators on $\widetilde{\Delta}\left(i_{j}\right)$ converge to the lowest eigenvalue of the Jacobi operator on $\widetilde{\Delta}$, which is negative. This implies $\widetilde{\Delta}\left(i_{j}\right)$ is unstable for $j$ large, which contradicts the stability of $\widetilde{M}_{i_{j}}$. Therefore $\Delta$ is stable. But a complete stable orientable surface $\Delta$ is a plane [5, 7] and so $M$ lies in a halfspace. By the Halfspace Theorem [17], $M$ must be a plane, which gives a contradiction. This contradiction proves $\Sigma$ is connected and, as we showed for $\Delta$ above, $\Sigma$ is stable. Since the boundary sphere of any ball $B(r)$ containing $\gamma$ in its interior must intersect $\widetilde{M}_{i_{j}}$ for all $j$ sufficiently large, $\Sigma$ must intersect $\partial B(r)$. This implies that $\Sigma$ is noncompact. If $\Sigma \cap \partial R_{1} \neq \gamma$, then the maximum principle implies $\Sigma \subset \partial R_{1}$ and must equal $\overline{\partial R_{1}-M_{1}}$ since $\Sigma$ is noncompact. Hence $\Sigma \cup \Sigma_{1}$ is a properly embedded piecewise smooth surface in $\mathbb{R}^{3}$ and so $\Sigma$ is orientable.

Thus, it suffices to prove that some subsequence $\widetilde{M}_{i_{j}}$ converges smoothly on compact subsets of $\mathbb{R}^{3}$ to a properly embedded minimal surface. To prove this it is sufficient to prove that on compact balls $B$ in $\mathbb{R}^{3}$, the surfaces $\widetilde{M}_{i} \cap B$ satisfy uniform 
curvature and area bounds. Let $\beta(i)$ denote the other boundary curve $\partial \widetilde{M}_{i}-\gamma$. Replace the surfaces $\widetilde{M}_{i_{j}}$ by smooth subdomains $N_{i_{j}}$, where $N_{i_{j}}$ is obtained from $\widetilde{M}_{i_{j}}$ by removing a smooth open subdomain of points in $\widetilde{M}_{i_{j}}$ of intrinsic distance approximately 1 from $\beta(i)$. Our first step in proving the existence of a convergent subsequence is to derive uniform curvature estimates for the family $\left\{N_{i_{j}}\right\}$ in all of $\mathbb{R}^{3}$.

Recall that Schoen [26] proved that there is a universal constant $c$ such that the Gaussian curvature $K$ on $\widetilde{M}_{i}$ satisfies

$$
|K(p)| \leq \frac{c}{(d(p))^{2}},
$$

where $d(p)$ is the intrinsic distance of $p$ to $\partial \widetilde{M}_{i}$. Thus to derive our uniform curvature estimates it remains to prove that the Gaussian curvature of the surfaces $\left\{N_{i}\right\}$ is uniformly bounded on some intrinsic $\varepsilon$-neighborhood of $\gamma, \varepsilon$ chosen independent of $i$.

Consider a small $\varepsilon$-neighborhood $F(\varepsilon)$ of $\gamma$ in $R_{1}$. There exists a small $\varepsilon_{0}$ such that for all positive $\delta<\varepsilon_{0}, A(\delta)=\partial F(\delta)-\partial R_{1}$ is an incompressible annulus in $R_{1}$ with nowhere zero mean curvature vectorfield pointing into $F(\delta)$ and the area of this annulus is at most $2 \pi \delta L$ where $L$ is the length of $\gamma .(A(\delta)$ is incompressible since $\gamma$ is homotopically nontrivial in $R_{1}$.) Fix such a number $\varepsilon_{0}$.

We want to prove that there is a choice of $\delta<\varepsilon_{0}$ such that for all $i$, the component of $N_{i} \cap F(\delta)$ containing $\gamma$ is an annulus. As a first step, we show that we can choose $\delta<\varepsilon_{0}$ so that every least-area disk $D \subset R_{1}$ with $\partial D \subset A(\delta)$ must be contained in $F(\delta)$. First suppose $D \subset F\left(\varepsilon_{0}\right)$. Then $D \subset F(\delta)$ by the maximum principle (applied to the family of surfaces $\left.A(t), \delta \leq t \leq \varepsilon_{0}\right)$. Suppose now that $D$ contains points outside of $A\left(\varepsilon_{0}\right)$. Then it contains some point of distance $\varepsilon_{0}-\delta$ from $\partial D$ and hence, by the monotonicity formula, $D$ has area at least $\pi\left(\varepsilon_{0}-\delta\right)^{2}$. Since $A(\delta)$ is incompressible in $R_{1}, \partial D$ bounds a disk in $A(\delta)$ of area less than $2 \pi \delta L$. Therefore, if $\delta$ is chosen to satisfy $\left(\varepsilon_{0}-\delta\right)^{2}>2 \delta L$, then $D$ does not have least area. This proves that $D \subset F\left(\varepsilon_{0}\right)$, after choosing $\delta$ so that $\left(\varepsilon_{0}-\delta\right)^{2}>2 \delta L$, and hence $D \subset F(\delta)$.

Since $\widetilde{M}_{i}$ is transverse to $A(t)$ for almost all small $t$, we can choose $\delta$ so that $A(\delta)$ is also transverse to all the $\widetilde{M}_{i}$. Let $\Delta(i)$ be the component of $\widetilde{M}_{i} \cap F(\delta)$ containing $\gamma$. Elementary three-manifold topology shows $\Delta(i)$ is an annulus if and only if it is incompressible in $F(\delta)$, since $\pi_{1}(F(\delta))=\mathbb{Z}$. Suppose $\Delta(i)$ is not incompressible in $F(\delta)$. Then there exists a homotopically nontrivial simple closed curve $\alpha(i)$ in $\Delta(i)$ that bounds a disk in $F(\delta)$. Since $\widetilde{M}_{i}$ is incompressible and $\Delta(i) \subset \widetilde{M}_{i}$, there exists a disk $D(i) \subset \widetilde{M}_{i}$ with boundary $\alpha(i)$. By our choice of $\delta, D(i) \subset F(\delta)$. This implies $D(i) \subset \Delta(i)$, which is impossible since $\Delta(i)$ is the component of $\widetilde{M}_{i} \cap F(\delta)$ containing $\gamma$. This contradiction proves there exists a $\delta$ such that $\Delta(i)$ is an annulus.

The areas of the least-area annuli $\Delta(i)$ are bounded by the sum of the areas of $A(\delta)$ and the area of $F(\delta) \cap\left(\Sigma_{1} \cup M\right)$, since $\partial \Delta(i)$ is the boundary of an annulus on this surface. This area bound implies that every subsequence $\Delta\left(i_{j}\right)$ contains a subsequence that converges smoothly in $F(\delta)-(\gamma \cup A(\delta))$. (See the proof of Theorem 2 in [23] for details on this convergence property.) Hildebrandt's boundary regularity theorem [15] states that such a least-area limit annulus is smooth along $\gamma$. A well-known consequence of Hildebrandt's proof of boundary regularity is that when a sequence $\{E(i)\}$ of smooth minimal surfaces converges to a smooth minimal 
surface $E$ and a component $\partial(i)$ of $\partial E(i)$ converges smoothly to a component $\partial$ of $\partial M$, then the convergence of these surfaces is smooth near $\partial$. Therefore, every subsequence $\Delta\left(i_{j}\right)$ contains a subsequence that converges smoothly in $F(\delta)$ $A(\delta)$ and this convergence is smooth along $\gamma$. Since every subsequence of the $\Delta(i)$ has a smoothly converging subsequence, the curvatures of all the $\Delta(i)$ are uniformly bounded. This completes the proof that the curvature of the family $\left\{N_{i}\right\}$ is uniformly bounded in $\mathbb{R}^{3}$.

Suppose $F$ is a compact surface in $\mathbb{R}^{3}$ satisfying

1. $F$ has one boundary component $\Gamma$ in $B\left(r_{1}\right)$ and the rest of its boundary components outside of the ball $B\left(r_{2}\right)$ where $r_{2}>r_{1}$.

2. The curvature of $\Gamma$ is bounded by a constant $C_{1}$.

3. The length of $\Gamma$ is bounded by a constant $C_{2}$.

4. The Gaussian curvature of $F$ is bounded from below by a constant $C_{3}$.

Let $E(t)$ be the area of the subdomain $F(t)$ of $F$ of intrinsic distance $t$ from $\Gamma$ for $t<\left(r_{2}-r_{1}\right)$. By comparison formulas with surfaces of constant Gaussian curvature $C_{3}$, there exist constants $K_{1}, K_{2}$ that depend on $C_{1}, C_{2}, C_{3}$ such that $E(t) \leq K_{1}+K_{2}^{t}$.

Suppose $\gamma \subset B\left(r_{1}\right)$. After discarding some of the surfaces $N_{i}$ and reordering, we may assume that the other boundary curves of $N_{i}$ lie outside $B(i)$. Then the above area estimate implies that the area $E(i, t)$ of the subdomain of intrinsic distance $t$ from $\gamma$ satisfies $E(i, t) \leq K_{1}+K_{2}^{t}$ for $t<i-r_{1}$, where $K_{1}, K_{2}$ depend on $\gamma$ and the earlier obtained uniform curvature estimate for $\left\{N_{i}\right\}$.

Suppose now that the areas of the family are not uniformly bounded on some large ball $B$. Since $E(i, t) \leq K_{1}+K_{2}^{t}$, for $t<i-r_{1}$, there exist a point $p \in B$ and a sequence of points $p\left(i_{j}\right) \in N_{i_{j}}$ converging to $p$ and such that $\operatorname{dist}\left(p\left(i_{j}\right), \partial N_{i_{j}}\right) \rightarrow \infty$ as $j \rightarrow \infty$, where dist is the intrinsic distance. By Schoen's curvature estimates [26], the surfaces $N_{i_{j}}$ are getting flatter and flatter in larger and larger disk regions $D\left(i_{j}\right)$ centered at $p\left(i_{j}\right)$ as $j \rightarrow \infty$. Hence, a subsequence of the $D\left(i_{j}\right)$ converges to a flat plane $P \subset R_{1}$ passing through $p$. By the Halfspace Theorem [17], $M$ must be a plane. This contradiction completes the proof of Assertion 3.1, which in turn completes the proof of Theorem 3.2.

\section{EXAMPles AND COUNTEREXAMPles}

We first describe three important classical examples of properly embedded minimal surfaces with one end and infinite genus. The first example is the triply-periodic minimal surface pictured in Image 1. It is called the Schwarz Primitive surface and was discovered independently by Schwarz and Riemann. In Images 2 and 3 appear two well-known examples of Scherk, one that is doubly-periodic and the other that is one-periodic. Scherk's doubly-periodic example is asymptotic in $\mathbb{R}^{3}$, as $x_{3} \rightarrow+\infty$, to the planes $P_{i}=\{(t, i, s) \mid t, s \in \mathbb{R}\}$ for $i \in \mathbb{Z}$ and asymptotic, as $x_{3} \rightarrow-\infty$, to the planes $Q_{i}=\{(i, t, s) \mid t, s \in \mathbb{R}\}$ for $i \in \mathbb{Z}$. Scherk's one-periodic surface is asymptotic, away from the $x_{2}$-axis, to the union of two orthogonal planes containing the $x_{2}$-axis.

Let $k \subseteq S^{3}$ be the Artin-Fox curve. Let $k^{\prime} \subset \mathbb{R}^{3}$ be the curve obtained by removing the wild point of the Artin-Fox curve from $k$ and $S^{3}$. Let $F$ be the boundary of a regular neighborhood of $k^{\prime}$. The closure of the component of the complement of $F$ that corresponds to the exterior of the regular neighborhood 


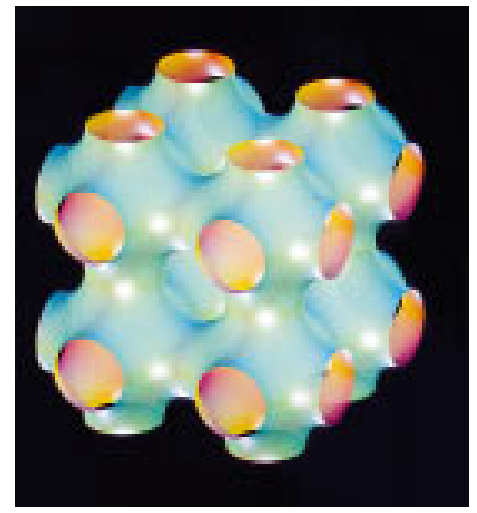

Image 1. Schwarz's Primitive Surface

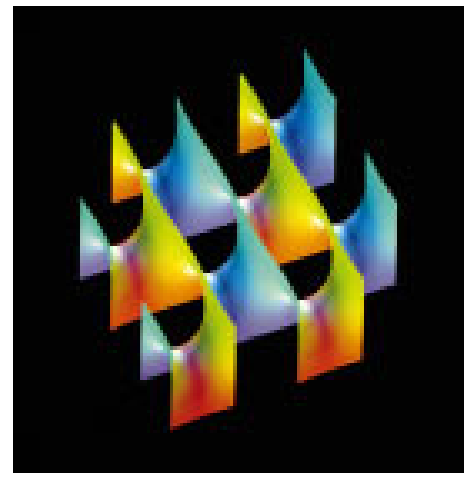

Image 2. Scherk's Doubly Periodic Surface

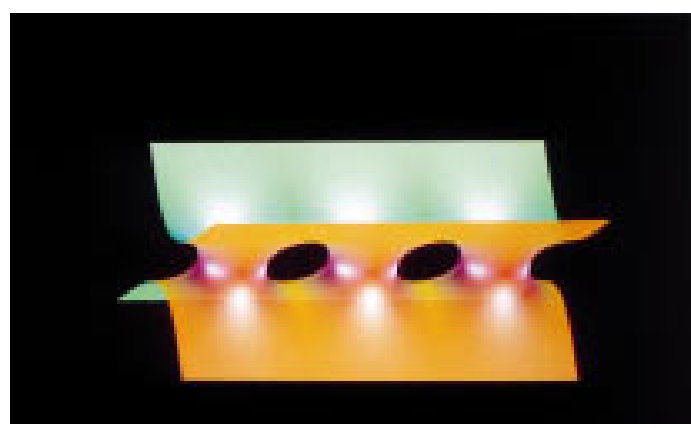

Image 3. Scherk's Singly Periodic Surface 


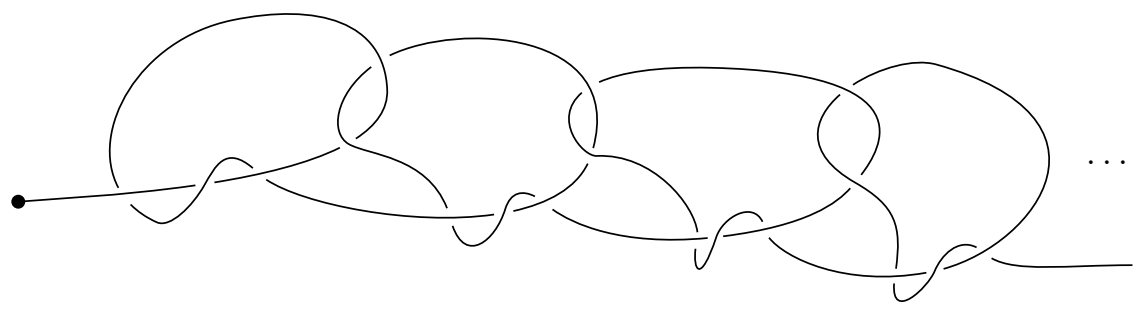

FIGURE 5

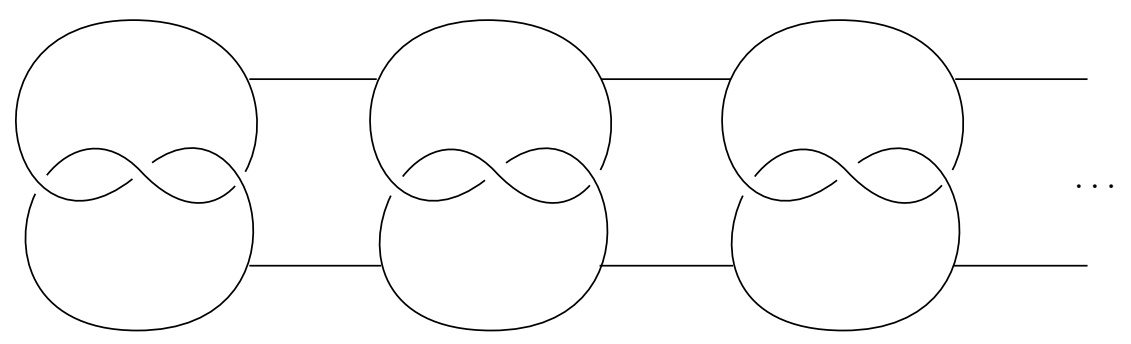

Figure 6

is a fake halfspace. Choose an infinite family of disks in $F$ that form a proper family. Replace each disk by an unknotted handle. The resulting surface $F^{\prime}$, pictured in Figure 5, is not a Heegaard surface. In this case it is possible to construct a nonproper standardizing sequence of disks for the wild side of $F$, where the boundary curves of the disks are proper.

An open handlebody is a three-manifold without boundary that is homeomorphic to an open regular neighborhood of some one-complex that is properly embedded in $\mathbb{R}^{3}$. In [19] it is proved that if $F \subseteq \mathbb{R}^{3}$ is a properly embedded surface in $\mathbb{R}^{3}$ and there exists $p \in \mathbb{R}^{3}$ such that the distance from $p$ on $F$ induces a Morse function having only index one and index zero critical points, then the connected components of the complement of $F$ in $\mathbb{R}^{3}$ are open handlebodies. This argument can be strengthened to show that if $W$ is the closure of a component of the complement of $F$, then $W$ can be embedded into a handlebody $H$ so that the complement of the image of $W$ in $H$ is a closed subset of the boundary of $H$. However, this is not enough to prove that $W$ is a handlebody. In Figure 6 we show a one-complex $X$ embedded in $\mathbb{R}^{3}$. Let $F$ be the boundary of a regular neighborhood of $X$. Let $W$ denote the closure of the component of the complement of $F$ corresponding to the exterior of the regular neighborhood. Notice that if $F$ was constructed carefully, then the distance from the point $p$ induces a Morse function on $F$ with exactly two index zero critical points and no index two critical points. However, $W$ is not a handlebody. In this case, $\pi_{i}(F) \rightarrow \pi_{1}(W)$ is injective (and surjective).

\section{ACKNOWLEDGEMENTS}

We would like to thank Bob Edwards for his interest in Theorems 1.1 and 1.2, as well as useful conversations related to their proof. We are also grateful to Jim Hoffman who produced the computer graphics images of Scherk's singly-periodic and doubly-periodic minimal surfaces and Schwarz's Primitive surface. Computer 
graphics images of these surfaces appear in Images 1, 2, and 3 in Section 4 . The production of these images were made with the facilities of GANG, the Center for Geometry, Analysis, Numerics and Graphics at the University of Massachusetts.

\section{REFERENCES}

1. M. Boileau and J. P. Otal, Sur les scindements de Heegaard du tore $\mathbb{T}^{3}$, Journal of Differential Geometry, 32, (1990), pp. 209-233. MR 91i:57006

2. F. Bonahon and J. P. Otal, Scindements de Heegaard des espaces Lenticulares, Ann. Scient. Éc. Norm. Sup. 16 (1983), no. 4, 451-466. MR 85c:57010

3. M. Callahan, D. Hoffman, and W. H. Meeks III, The structure of singly-periodic minimal surfaces, Inventiones Math. 99 (1990), 455-481. MR 92a:53005

4. A. J. Casson and C. McA. Gordon, Reducing Heegaard splittings, Topology and Its Applications 27 (1987), 275-283. MR 89c:57020

5. M. do Carmo and C. K. Peng, Stable minimal surfaces in $\mathbb{R}^{3}$ are planes, Bulletin of the AMS 1 (1979), 903-906.

6. D. Fischer-Colbrie, On complete minimal surfaces with finite Morse index in 3-manifolds, Inventiones Math. 82 (1985), 121-132. MR 87b:53090

7. D. Fischer-Colbrie and R. Schoen, The structure of complete stable minimal surfaces in 3manifolds of non-negative scalar curvature, Comm. on Pure and Appl. Math. 33 (1980), 199-211. MR 81i:53044

8. M. Freedman, J. Hass, and P. Scott, Least area incompressible surfaces in 3-manifolds, Inventiones Math. 71 (1983), 609-642. MR 85e:57012

9. C. Frohman, Minimal surfaces and Heegaard splittings of the three-torus, Pacific Journal of Math. 124 (1986), 119-130. MR 87j:57011

10. - The topological uniqueness of triply-periodic minimal surfaces in $\mathbb{R}^{3}$, Journal of Differential Geometry 31 (1990), 277-283. MR 92e:53008

11. _ Heegaard splittings of the three-ball, Topology '90, Proceedings of the Special Semester on Low Dimensional Topology, Ohio State University, De Gruyter, Berlin, 1992. MR 94b:57015

12. C. Frohman and J. Hass, Unstable minimal surfaces and Heegaard splittings, Inventiones Math. 95 (1989), 529-540. MR 90e:57028

13. R. Hardt and L. Simon, Boundary regularity and embedded minimal solutions for the oriented Plateau problem, Annals of Math. 110 (1979), 439-486. MR 81i:49031

14. J. Hempel, Three manifolds, Annals of Math Studies, no. 72, Princeton University Press, Princeton, NJ, 1976. MR 54:3702

15. S. Hildebrandt, Boundary behavior of minimal surfaces, Archive Rational Mech. Anal. 35 (1969), 47-81. MR 40:1901

16. D. Hoffman, H. Karcher, and F. Wei, Adding handles to the helicoid, Bulletin of the AMS, New Series 29 (1993), no. 1, 77-84. MR 94g:53004

17. D. Hoffman and W. H. Meeks III, The strong halfspace theorem for minimal surfaces, Inventiones Math. 101 (1990), 373-377. MR 92e:53010

18. H. B. Lawson, The unknottedness of minimal embeddings, Inventiones Math. 11 (1970), 183-187. MR 44:4651

19. W. H. Meeks III, The topological uniqueness of minimal surfaces in three-dimensional Euclidean space, Topology 20 (1981), 389-410. MR 82h:53010

20. $\quad$ The theory of triply-periodic minimal surfaces, Indiana University Math. Journal 39 (1990), no. 3, 877-936. MR 92e:53012

21. W. H. Meeks III and H. Rosenberg, The maximum principle at infinity for minimal surfaces in flat three-manifolds, Commentari Mathematici Helvetici 65 (1990), 255-270. MR 91d:53011

22. W. H. Meeks III, L. Simon, and S. T. Yau, The existence of embedded minimal surfaces, exotic spheres and positive Ricci curvature, Annals of Math. 116 (1982), 221-259.

23. W. H. Meeks III and S. T. Yau, The classical Plateau problem and the topology of threedimensional manifolds, Topology 21 (1982), no. 4, 409-442. MR 84g:53016

24. - The existence of embedded minimal surfaces and the problem of uniqueness, Math. Z. 179 (1982), 151-168. MR 83j:53060

25. - The topological uniqueness of complete minimal surfaces of finite topological type, Topology 31 (1992), no. 2, 305-316. MR 93g:57037 
26. R. Schoen, Estimates for stable minimal surfaces in three dimensional manifolds, Annals of Math. Studies, vol. 103, Princeton University Press, 1983. MR 86j:53094

27. _ Uniqueness, symmetry, and embeddedness of minimal surfaces, Journal of Differential Geometry 18 (1983), 791-809. MR 85f:53011

28. G. P. Scott and T. Wall, Homological group theory, London Math Society Lecture Notes, no. 36, Cambridge University Press, 1979. MR 81 m:57002

29. L. Simon, Lectures on geometric measure theory, Proceedings of the Center for Mathematical Analysis (Canberra, Australia), vol. 3, Australian National University, 1983. MR 87a:49001

30. J. Singer, Three-dimensional manifolds and their Heegaard diagrams, Transactions of the AMS 35 (1933), 88-111.

31. F. Waldhausen, Heegaard-zerlegungen der 3-sphäre, Topology 7 (1968), 195-203. MR 37:3576 